\title{
II. On motion in a periodic field of force
}

\section{C.V. Raman M.A.}

To cite this article: C.V. Raman M.A. (1915) II. On motion in a periodic field of force, Philosophical Magazine Series 6, 29:169, 15-27, DOI: 10.1080/14786440108635276

To link to this article: http://dx.doi.org/10.1080/14786440108635276

$$
\text { 册 Published online: } 08 \text { Apr } 2009 .
$$

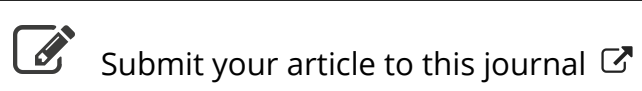

\footnotetext{
Џll Article views: 6
}

Q View related articles $\sqsubset$ 
The conditions for maximum potential are the same when a rapid interruptor is employed as for the slow break used in the above experiments. They also hold whether the primary current is supplied by a small storage-battery, a 100-volt battery, or the 200-volt mains. Plate I. fig. 8 shows the course of the secondary potential at two successive "brealss" effected by a motor mercury interruptor. 'The effect of the small potential at the "make" is also noticeable. On this occasion the 100-volt battery was used to supply the primary current, the total (steady-current) resistance in the primary circuit, being 11 ohms. The greatest spark-length was $18.4 \mathrm{~cm}$., and the mean primary current, as indicated by an amperemeter in the primary circuit, was $\cdot 3$ ampere. The current immediately before interruption would, however, be over 2 amperes.

Bangor, November 1914.

II. On Motion in a Periodic Field of Force. By C. V. RAMAX, M.A.*

[Plate II.]

Vibrations maintained by a Periodic Field of Force.

THE experimental study of the motion of a dynamical 1 system in a periodic field of force leads to results of quite exceptional interest. One aspect of the problem, $i . e$. the oscillatory motion of the system about a position of equilibrium in the field, has some affinities to the case of vibrations maintained by a variable spring which I have dealt with in my previously published work, but the two classes of investigations lead to results which differ from one another, yet are related in a most remarkable way. By experimenting on stretched strings subjected to a variable tension, I showed that a normal variation of spring will enable the oscillations of the system to be maintained, when the frequency of these oscillations is sufficiently nearly equal to $\frac{1}{2}$ of, or $\frac{2}{2}$ times, or $\frac{3}{2}$ times, or $\frac{4}{2}$ times, \&c. the frequency of variation of the spring, these ratios forming an ascending series $\dagger$. By experiments on the vibrations of a body about a position of equilibrium in a periodic field of force (to be described below), I have shown that the frequency of the oscillations maintained may be equal to, or half of, or one-third, or onefourth, \&c. of the frequency of the field; in other words, it

* Communicnted by the Author.

+ See Phil. Mag. Oct. 1912, "The Maintenance of Forced Oscillations of a New Type." 
may be any one of a descending series of sub-multiples of the frequency of the field. It appears, in fact, that we have here an entirely new class of resonance-vibrations. It will be noticed that if the two series referred to above are both written in the same order of descending magnitudes of frequency, thus,

$$
\begin{aligned}
& \frac{6}{2}, \frac{5}{2}, \frac{4}{2}, \frac{3}{2}, \frac{2}{2}, \frac{1}{2}, \\
& \frac{1}{T}, \frac{1}{2}, \frac{1}{3}, \frac{1}{4}, \frac{1}{3}, \frac{1}{6},
\end{aligned}
$$

the last two terms of the first series, and the first two of the second series coinside, and these two are to some extent typical of the rest. For, as I have shown in a previous publication *, the 1st, the 3rd, the 5th, and the odd types generally in the first series bear a family resemblance to each other, giving symmetrical vibration curves. The 2nd, the 4th, and the other even types similarly resemble each other in giving markedly asymmetrical vibration curves. Since the first term in the ascending series is the 2nd in the descending series, we may expect that the $2 \mathrm{nd}, 4$ th, 6 th, \&c. in the latter would give analogous types of motion, and that similarly the 1st, 3rd, 5th, \&c. would show resemblances amongst each other. These points will be dealt with more fully as we proceed.

The vibrations studied which form the subject of this section were those of the armature-wheel of a synchronous motor of the attracted-iron type, about a position of equilibrium in the magnetic field produced by an intermittent current circulating in the coils of an electromagnet. The phonic wheel or synchronous motor devised by La Cour and Lord Rayleigh is, as is well known, of great service in acoustical investigations. In my own work on vibrations and their maintenance, it has been of considerable assistance. Apart, however, from the various uses of the instrument in different branches of Physics and in Applied Electricity, it possesses much intrinsic interest of its own as an excellent illustration of the dynamies of a system moving in a periodic field of force, and the present paper deals almost entirely with experiments carried out by its aid and with its applications to the study of vibrations.

The instrument used by me was supplied by Messrs. Pye \& Co., of Cambridge, and has given entire satisfaction. The motor consists of a wheel of soft iron mounted on an axis with ball-bearings between the two poles of an electromagnet placed diametrically with respect to it. The wheel has thirty

* See Plysical Reriew, Dec. 1912. 
teeth, and when a direct current is passed through the electromagnet, sets itself rigidly at rest with a pair of teeth at the ends of a diameter opposite the two poles of the electromagnet. The equilibrium under such conditions is of course thoroughly stable, and, in fact, the wheel possesses a fairly high frequency of free angular oscillation for displacements from this position of rest, and any motion set up by such displacement rapidly dies out, apparently on account of Foucault currents induced in the iron by the motion. This, in general, is also true when an intermittent current supplied by a fork-interrupter is used to excite the electromagnet, except however in certain cases, when it is observed that the equilibrium becomes unstable of its own accord and the wheel settles down into a state of steady vigorous vibration about the line of equilibrium : or that an oscillation of sufficient amplitude once started maintains itself for an indefinitely long period.

An optical method can be conveniently used to study the frequency and the phase of the oscillations of the armaturewheel maintained in the manner described above. A narrow pencil of light is used, which first suffers reflexion at the surface of a small mirror attached normally to one of the prongs of the fork-interrupter furnishing the intermittent current, and then falls upon a second similar mirror attached to the axle of the armature-wheel parallel to its axis of rotation. The apparatus is so arranged that the angular deflexions produced by the oscillations of the fork and the wheel are at right angles to each other, and the pencil of light which falls upon a distant screen, or which is focussed on the ground-glass of the photographic camera, is seen to describe a Lissajous figure from which the frequency, and the phase-relations between the oscillations of the fork-interrupter and of the armature-wheel, can be readily ascertained. It is then observed that the period of the vibration of the armaturewheel is equal to, or twice, or thrice, or four times, \&c. the period of the fork : in other words, the frequency is equal to or $\frac{1}{2}$ of or $\frac{1}{3}$ or $\frac{1}{4}$ or $\frac{1}{5}$ or $\frac{1}{6}$ that of the fork.

In making the experiments, the motor-wheel is relieved of the large stroboscopic disk that is usually mounted upon it, and in working down the series, the adjustment of frequency is secured by suitably loading the wheel. The fine adjustment for resonance is effected by altering the current passing through the interrupter with the aid of a rheostat, and if necessary by regulating the contact-maker on the fork. Any oscillation of the wheel, when started, dies away except in the cases referred to above ; in other words, no frequencies

Plit. Mag. S. 6. Vol, 29. No. 169. Jan. 1915. 
intermediate between those of the series are maintained. In obtaining the 1st case, in which the oscillations of the wheel and the fork are in unison, it is generally found necessary to increase the "spring" of the wheel by passing a steady direct current through the electromagnet of the motor from a cell connected in parallel, in addition to the intermittent current flowing in the same direction from the interrupter circuit.

It is found that the Lissajous figures for the 1st, 3rd, and 5th cases are distinctly asymmetrical in character, the $3 \mathrm{rd}$ being markedly so. The 2 nd, 4 th, and 6 th types are quite symmetrical. I his, it will be remembered, was what was anticipated above, and in fact the 1st, 3rd, and 5th types differ rather markedly in their behaviour from the 2nd, 4th, and 6 th types. These latter are maintained with the greatest ease, while the former, particularly the 5 th, are not altogether so readily maintained. In fact it is found advantageous, in order to maintain the 5th type steadily, to load the wheel somewhat unsymmetrically and to put it a little out of level, in order to allow the oscillations to take place about an axis slightly displaced from the line joining the poles of the electromagnet.

It is noticed also that the lower frequencies of vibration have much larger amplitudes. This, I would attribute principally to the greatly reduced damping at the lower frequencies owing to the slower motion, the larger masses, and the weaker magnetic fields employed.

We are now in a position to consider the mathematical theory of this class of maintained vibrations. To test the correctness of my theoretical work, I have prepared a series of photographs of the simultaneous vibration-curves of the fork and of the armature-wheel, which are reproduced as figs. 1 to $6, \mathrm{Pl}$. II. These curves were obtained by the usual method of recording the vibrations optically on a moving photographic plate, it being so arranged that the directions of movement of the two representative spots of light on the plate lie in the same straight line. The upper curve in each case shows the maintained vibration of the armature-wheel. The lower represents that of the forkinterrupter. The frequency of the former, it will be seen, is 1 or $\frac{1}{2}$ or $\frac{1}{3}$ or $\frac{1}{4}$ or $\frac{1}{5}$ or $\frac{1}{6} \times$ that of the latter. The precise features of the vibration-curve noticed in each case will be referred to below, in connexion with the mathematical discussion.

The equation of motion of a system with one degree of freedom moving in a periodic field of force, and subject also 
to the usually assumed type of viscous resistance, may be written in the following form,

$$
\ddot{\mathrm{U}}+k \dot{\mathrm{U}}+2 a f(t) \mathrm{F}(\mathrm{U})=0, \quad \cdot . .
$$

where $F(U)$ gives the distribution of the field, $f(t)$ its variability with respect to time, and $2 a$ is a constant. If we are dealing with oscillations about a position that would be one of stable equilibrium if the field were constant. $F(U)$ may as an approximation be put equal to $U$. We then have

$$
\ddot{\mathrm{U}}+k \dot{\mathrm{U}}+2 a f(t) \mathrm{U}=0 . . . \quad . \quad .
$$

In the experiments described above, the periodicity of $f(t)$ is the same as that of the intermittence of the exciting current. If an alternating current had been used, the frequency of $f(t)$ would have been double that of the alternations. In any case we may write

$$
\begin{aligned}
a f(t) & =a_{1} \sin n t+a_{2} \sin 2 n t+a_{3} \sin 3 n t+\& c . \\
& +b_{0}+b_{1} \cos n t+b_{2} \cos 2 n t+b_{3} \cos 3 n t+\& c . .
\end{aligned}
$$

Since $U$ is shown to be periodic by experiment, we may write

$$
\begin{aligned}
\mathrm{U}= & \mathrm{A}_{1} \sin p t+\mathrm{A}_{2} \sin 2 p t+\mathrm{A}_{3} \sin 3 p t+\& \mathrm{c} . \\
& +\mathrm{B}_{0}+\mathrm{B}_{1} \cos p t+\mathrm{B}_{3} \cos 2 p t+\mathrm{B}_{3} \cos 3 p t+\& \mathrm{c} .
\end{aligned}
$$

As a typical example of the even types of maintenance, we may take the cases in which $n=4 p$. We have

$$
\begin{aligned}
a f(t) & =a_{1} \sin 4 p t+a_{2} \sin 8 p t+a_{3} \sin 12 p t+\& \mathrm{c} . \\
& +b_{0}+b_{1} \cos 4 p t+b_{2} \cos 8 p t+b_{3} \cos 12 p t+\& \mathrm{c} .
\end{aligned}
$$

In this case, and also in the case of the second, sixth, and in fact in all the even types of maintenance, we find that the quantities $A_{2}, A_{4}, A_{6}$, \&c., and $B_{0}, B_{2}, B_{4}$, \&c., do not enter into the equations containing $A_{1}$ and $B_{1}$. We therefore write them all equal to zero. The significance of this is that with the even types of vibration maintained by a periodic field of force, the even harmonics are all absent from the maintained motion. This result is fully verified by a reference to the vibration-curves of the 2 nd, 4 th, and 6 th types shown in figs. 2,4 , and $6, \mathrm{Pl}$. II. It will be seen that the vibratory motion of the armature-wheel has that type of symmetry so familiar in alternating current curves, in which all the even harmonics are absent. In other words, the image of onehalf of the curve above the zero axis, as seen by reflexion in a mirror placed parallel to this axis, is exactly similar to the other half below it. 
Substituting now the odd terms alone left on the righthand side of (4), for $U$ in equation (2), we have the following series of equations:-

$$
\begin{aligned}
& -\left(b_{0}-p^{2}\right) \mathrm{A}_{1}+k p \mathrm{~B}_{1}=-b_{1} \mathrm{~A}_{3}+a_{1} \mathrm{~B}_{3}+b_{1} \mathrm{~A}_{5}-a_{1} \mathrm{~B}_{5}-\& c .+\& c_{.} \\
& -\left(b_{0}-p^{2}\right) \mathrm{B}_{1}-k p \mathrm{~A}_{1}=a_{1} \mathrm{~A}_{3}+b_{1} \mathrm{~B}_{3}+a_{1} \mathrm{~A}_{5}+b_{1} \mathrm{~B}_{5}+\& c .+\& c . \\
& -\left(b_{0}-9 p^{2}\right) \mathrm{A}_{3}+3 k p \mathrm{~B}_{3}=-b_{1} \mathrm{~A}_{1}+a_{1} \mathrm{~B}_{1}-b_{2} \mathrm{~A}_{5}+a_{2} \mathrm{~B}_{5}+\& c .-d c . \\
& -\left(b_{0}-9 p^{2}\right) \mathrm{B}_{3}-3 k p \mathrm{~A}_{3}=a_{1} \mathrm{~A}_{1}+b_{1} \mathrm{~B}_{1}+a_{2} \mathrm{~A}_{5}+b_{2} \mathrm{~B}_{5}+\& \mathrm{c} .+\& c .(6)
\end{aligned}
$$

and so on.

Evidently, the possibility of this being a consistent set of convergent equations depends upon the suitability of the values assigned to the constants $k, p, b_{0}, a_{1}, b_{1}$, \&c.

It is not possible here to enter into a complete discussion of the solution of these equations. One point is, however, noteworthy. From the first two of the set of equations given above, it will be seen that such of the harmonics in the steady motion of the system as are present serve as the vehicles for the supply of the energy requisite for the maintenance of the fundamental part of the motion. Paradoxically enough, the frequency of none of these harmonics is the same as that of the field.

We now proceed to consider the odd types of vibration, i. e. the 1st, the 3rd, \&c. Taking the 3rd as a typical case, we put $n=3 p$ and get

$$
\begin{aligned}
& a f(t)=a_{1} \sin 3 p t+a_{2} \sin 6 p t+a_{3} \sin 9 p t+\& \mathrm{c} . \\
& \quad+b_{0}+b_{1} \cos 3 p t+b_{2} \cos 6 p t+b_{3} \cos 9 p t+\& \mathrm{c} .
\end{aligned}
$$

Substituting (4) and (7) in equation (2) and equating the coefficients of sine and cosine terms of various periodicities to zero, we find that the quantities $A_{3}, A_{6}, A_{9}, \& c$. and $B_{0}$, $B_{3}, B_{6}, B_{9}$ \&c., do not enter into the equations containing $A_{1}$ and $B_{1}$. We therefore write them all equal to zero. The significance of this is that the maintained motion contains no harmonics the frequency of which is the same as, or any multiple of the frequency of the periodic field of force. This remarkable result is verified by a reference to fig. 3 , Pl. II. from which it is seen, that the vibration curve is roughly similar to that of the motion of a trisection point of a string bowed near the end, the 3rd component, the 6th, the 9th, \&c., being absent at the point of observation. 
We then obtain the following set of relations by substitution :

$-\left(b_{0}-p^{2}\right) \mathrm{A}_{1}+k p \mathrm{~B}_{1}=-b_{1} \mathrm{~A}_{2}+a_{1} \mathrm{~B}_{2}+b_{1} \mathrm{~A}_{4}-a_{1} \mathrm{~B}_{4}-\& c$.

$-\left(b_{0}-p^{2}\right) \mathrm{B}_{1}-k p \mathrm{~A}_{1}=a_{1} \mathrm{~A}_{2}+b_{1} \mathrm{~B}_{2}+a_{1} \mathrm{~A}_{4}+b_{1} \mathrm{~B}_{4}+\& \mathrm{c}$.

$-\left(b_{1}-4 p^{2}\right) \mathrm{A}_{2}+2 k p \mathrm{~B}_{2}=-b_{1} \mathrm{~A}_{1}+a_{1} \mathrm{~B}_{1}+b_{1} \mathrm{~A}_{5}-a_{1} \mathrm{~B}_{5}+\& c$.

$-\left(b_{0}-4 p^{2}\right) \mathrm{B}_{2}-2 k p \mathrm{~A}_{2}=a_{1} \mathrm{~A}_{1}+b_{1} \mathrm{~B}_{1}+a_{1} \mathrm{~A}_{5}+b_{1} \mathrm{~B}_{5}+\& c$.

and so on.

It must be remembered that these relations are all only approximate, as $F(U)$ in general contains powers of $U$ higher than the first which we have neglected, and which no doubt must be taken into account in framing a more complete theory. The general remarks made above with reference to equation (6) apply here also.

The exact character of the vibratory motion maintained by the periodic field of force in any case, depends upon the form of the functions $F(\mathrm{U})$ and $f(t)$ which determine respectively the disposition of the field and its variability with respect to time. One very simple and important form of $f(t)$ is that in which the field is of an impulsive character, in other words is of great strength for a very short interval of time comprised in its period of variation, and during the rest of the period is zero or nearly zero. Such a type of variation is not merely a mathematical possibility. In actual experiment, when a fork-interrupter is used to render the current passing through the electromagnet intermittent, the magnetization of the latter subsists only during the small fraction of the period during which the current flows and at other times is practically zero. When the current is flowing the acceleration is considerable: at other times, the acceleration is nearly zero, and the velocity practically constant. These features are distinctly shown in all the vibration-curves (except those of the first type) reproduced in Pl. II., the sudden bends in the curves corresponding roughly to the extreme outward swings of the fork, i.e. to the instants when the magnetizing current was a maximum. It seems possible that a simpler mathematical treatment than that given above might be sufficient to discuss the phenomena of the maintenance of vibrations by a periodic field of force when the periodicity of the field is of the "impulsive" type ; in other words, when the dynamical system is subject to periodic impulsive "springs," one, two, three or more of which occur at regular intervals during each complete period of the vibration of the system.

These experiments on vibrations maintained by a periodic 
field of force are very well suited for lecture demonstration, as the Lissajous figures obtained by the method described above can be projected on the screen on a large scale, and form a most convincing demonstration of the fact that the frequency of the maintained motion is an exact sub-multiple of the frequency of the exciting current.

\section{On Synchronous Rotation urder Simple Eacitation.}

It is well known that with an intermittent current passing through its electromagnet, the synchronous motor can maintain itself in "tuniform" rotation, when for every period of the current, one tooth in the armature-wheel passes each pole of the electromagnet. In other words, the number of teeth passing per second is the same as the frequency of the intermittent current. From a dynamical point of view it is of interest, therefore, to investigate whether the motor could run itself successfully at any speeds other than the "synchronous" speed. Some preliminary trials with the motor unassisted by any independent driving proved very encouraging. The phonic wheel I have is mounted on ballbearings, and runs very lightly when the large stroboscopic disk usually kept fixed upon it is taken off, and there is no current passing through the motor. When a cortinuous or intermittent current is flowing through the motor, the latter does not, however, run very lightly, being subject to very large electromagnetic damping, apparently due to Foucault currents in the iron. In the preliminary trials, however, I found that, using the intermittent unidirectional current from an interrupter-fork of frequency 60 , the motor could run successfully of itself at half the synchronous speed, $i . e$. with 30 teeth passing per second. It of course ran very well at the usual synchronous speed, $i$. e. with 60 teeth passing per second. By increasing the speed, it was found that the motor could also run well of itself at double the synchronous speed, $i$. $e$. with 120 teeth passing per second. Using an interrupter-fork of low frequency (23.5 per second) the motor, it was found, could also run of itself at triple the synchronous speed. No certain indication was, however, obtained of the intermediate speeds, $i$. e. $1 \frac{1}{2}$ and $2 \frac{1}{2}$ times respectively the synchronous speed.

To test these points, therefore, independent driving was provided. This was very satisfactorily obtained by fixing a small vertical water-wheel to the end of the axis of the motor and directing a jet of water against it. The waterwheel was boxed in to prevent any splashing of water on the observer. By regulating the tap leading up to the jet, the 
velocity of the latter could be adjusted. The speed of the phonic wheel was ascertained by an optical method, $i$. $e$. by observing the rim of the wheel as seen reflected in a mirror attached to the prong of the interrupter-fork. When the motor "bites," the pattern seen becomes stationary and remains so for long intervals of time or even indefinitely, and the speed of the wheel can be inferred at once from the nature of the pattern seen.

It was found in these trials that the motor could "bite" and run at the following speeds. (Frequency of interrupter 60 per sec.)

(a) $\frac{1}{2}$ the synchronons speed: stationary pattern of rim of moving wheel seen as a single sine-curve: wavelength $\frac{1}{2}$ the interval between teeth. Number of teeth passing electromagnet per second $=30$.

(b) Synchronous speed: stationary pattern of rim of moving wheel seen as a sine-curve, wave-length = interval between teeth. Number of teeth passing electromagnet per second $=60$.

(c) $1 \frac{1}{2}$ times the synchronons speed : stationary pattern of rim of wheel seen as three interlacing waves. Number of teeth passing electromagnet per second $=90$.

(d) 2 times the synchronous speed: stationary pattern seen as two interlacing curves. Number of teeth passing per second $=120$.

(e) $2 \frac{1}{2}$ times the synchronous speed : this was only obtained with difficulty. Number of teeth passing per second $=150$.

(f) 3 times the synchronous speed: stationary pattern seen as three interlacing curves. Very satisfactory running. Number of teeth per second $=180$.

(g) 4 times the synchronous speed: stationary pattern seen as 4 interlacing curves. Number of teeth per second $=240$.

(h) 5 times the synchronous speed : stationary pattern seen as 5 interlacing curves. Number of teeth per second $=300$.

The outstanding fact of observation is that while speeds which are equal to the "synchronous" speed or any integral multiple of it are readily maintained, only the first two or three members of the other series (i.e. having ratios $\frac{1}{2}, 1 \frac{1}{2}$, \&c. to the synchronons speed) can be obtained, and the "grip" of the wheel by the periodic magnetic forces, $i$. $e$. the stability of the motion, is hardly so great as in the integral 
series. This fact may be explained in the following general manner.

We may assume, to begin with, that the independent driving is less powerful than that required to overcome resistances, so that the wheel is a little belind the correct phases. In the case of the integral series, one or two or more teeth pass for every intermittence of the current, the wheel being in the same relative position, whatever this may be, to the electromagnet, at each phase of maximum magnetization of the latter. This is not, however, the case with the fractional speeds. It is only at every alternate phase of maximum magnetization that the wheel assumes the same position (whatever this may be) relative to the electromagnet. At the intermediate phases, it is displaced throngh a distance approximately equal to half the interval between the teeth. Whereas with the integral series, every phase of maximum magnetization assists the rotation, in the fractional series the wheel is alternately assisted and retarded by the successive phases of maximum magnetization, and it is the net effect of assistance that we perceive, this being of course comparatively small.

As the synchronous, half-synchronous, and double-synchronous speeds can all be readily maintained without independent driving, they can be very effectively exhibited as lecture experiments by lantern projection in the following way. The synchronous motor (which is quite small and light when the stroboscopic disk is removed) is placed on the horizontal stage of the lantern and the rim of the wheel is locussed on the screen. In front of the projection prism, where the image of the source of light is formed, is placed the fork-interrupter with the necessary device for intermittent illumination fitted to its prongs. When these are set into vibration and the synchronous motor is set in rotation, the "pattern" corresponding to the maintained speed becomes visible on the screen, and the effect of reversing the direction of rotation can also be demonstrated.

We now proceed to discuss the mathematical theory of the maintenance of uniform rotation in each of these cases. The first step is obviously to show that with the assumed velocity of rotation, the attractive forces acting on the disk communicate sufficient energy to it to balance the loss due to frictional torces. Taking the line joining the poles as the axis of $x$, the position of the wheel at any instant may be defined by the angle $\theta$ which a diameter of the wheel passing through a given pair of teeth makes with the axis of reference. If $n$ is the number of teeth in the wheel, the couple acting 
on the latter for any given field strength at the poles is obviously a periodic function of $n \theta$ which vanishes when $\theta=\frac{2 \pi r}{n}$, and also when $\theta=\frac{2 \pi\left(r+\frac{1}{2}\right)}{n}$, where $r$ is any integer.

We therefore write

Couple $=$ Field strength $\times\left[a_{1} \sin n \theta+a_{2} \sin 2 n \theta+a_{3} \sin 3 n \theta+\&\right.$ c. $]$

$=$ Field stren $g$ th $\times f(n \theta)$ say,

where the terms $a_{1}, a_{2}, a_{3}$, \&c. rapidly diminish in amplitude. It will be seen that the cosine terms are absent. Since the field strength is periodic, we may write the expression for the couple acting on the wheel thus

$$
\begin{aligned}
\text { Couple } & =\mathrm{L} f(n \theta)\left[b_{1} \sin \left(p t+\epsilon_{1}\right)+b_{2} \sin \left(2 p t+\epsilon_{2}\right)+\& c .\right] \\
& =\mathrm{L} f^{\prime}(n \theta) \mathrm{F}(t), \text { say. }
\end{aligned}
$$

The work done by the couple in any number of revolutions

$$
=\int \mathrm{L} f(n \theta) \mathbf{F}(t) d t .
$$

It is obvious that this integral after any number of complete revolutions is zero, except in any of the following cases, when it has a finite value proportional to and increasing with $t ; i$. e. when

$$
n \theta=p t \text { or } 2 p t \text { or } 3 p t \text { or } 4 p t \text { and so on, }
$$

or when

$$
2 n \theta=p t \text { or } 2 p t \text { or } 3 p t \text { or } 4 p t \text { and so on, }
$$

or when

$$
3 n \theta=p t \text { or } 2 p t \text { and so on. }
$$

It is therefore a necessary but not, of course, always a sufficient condition for uniform rotation to be possible that one or more of the above relations should be satisfied. The first series corresponds to the synchronous speed and multiples of the synchronous speed. These have been observed experimentally by me up to the fifth at least. The second series includes the above and also the half-synchronous speed and odd multiples of the same. These latter have also been observed by me up to the fifth odd multiple. Since $a_{2}$ is much smaller than $a_{1}$, the relative feebleness of the maintenance of the half-speeds observed in experiment will readily be understood.

The third series has not so far been noticed in experiment. 
It is obvious that the maintaining forces in it should be excessively feeble compared with the first or the second. Perhaps experiments with interrupter-forks of higher frequencies and independent driving of the motor may succeed in showing the existence of controlled rotation-speeds at these ratios.

\section{Combinational Rotation-speeds under Double Excitation.}

When the electromagnet of the synchronous motor is excited simultaneously by the intermittent currents from two separate interrupter-forks having different frequencies, maintenance of uniform rotation is possible not only at the various speeds related to the synchronous speeds due to either of the intermittent currents acting by itself, but also at speeds related jointly to the frequencies of the two currents.

The preliminary experiments on this point were made without the assistance of any independent driving of the motor, and it was found at once that differential rotation of the motor was easily maintained, the number of teeth passing per second being equal to the difference of the frequencies of the two interrupter-forks.

When the "differentially" revolving wheel was examined by reflexion in mirrors attached to the prongs of the two interrupter-forks, it was found that the patterns seen in neither of them were stationary. They were found to be moving steadily forward or backward with a definite speed, with occasional slight to and fro oscillations superposed thereon. This continuous rotation of the patterns seen was obviously due to the fact that the frequencies of the forks and their difference did not bear any simple arithmetical ratios to each other, and it enabled a rotation-speed maintained by joint action to be distinguished by mere inspection from one maintained by either of the two currents separately.

Using this optical method, and assisting the rotation of the motor with independent driving by a water-motor, various other combinational speeds were found to be maintained. Of these, the most powerfully and steadily maintained was the simple summational rotation. The summationals and differentials of the second series, $i$. $e$. those in which the balf-frequencies of the fork enter, were also noticed. The rotation-speeds were determined by actual counting and a stop-watch.

The mathematical theory of these combinational speeds is very similar to that given for the case of excitation by one periodic current. For the field strength in this case is also 
a periodic function of the time, and the function $F(t)$ which expresses its value at any instant may be expanded in the following form

$$
\mathrm{F}(t)=a \Sigma \Sigma b \sin \left[\left(r p_{1} \pm s p_{2}\right) t+\mathrm{E}\right],
$$

where $p_{1} / 2 \pi$ and $p_{2} / 2 \pi$ are the frequencies of the two interrupters, and $r, s$ are any two positive integers. Using the same notation as before, we find that in any complete number of revolutions, a finite amount of energy proportional to the time is communicated to the wheel only in any one of the following sets of cases:

$$
\begin{array}{rlrl}
n \theta & =\left(r p_{1} \pm s p_{2}\right) t, \\
\text { or } & 2 n \theta & =\left(r p_{1} \pm s p_{2}\right) t, \\
\text { or } & 3 n \theta & =\left(r p_{1} \pm s p_{2}\right) t,
\end{array}
$$

and so on.

The cases actually observed in which rotation is maintained fall within the first two of the sets given above.

\section{Summary and Conclusions.}

The vibrations of a dynamical system maintained by a periodic field of force have been investigated experimentally and theoretically, and it is shown that they form a new class of resonance-vibrations, in which the frequency of the maintained motion is any sub-multiple of the frequency of the exciting force. The possible speeds of synchronous rotation of a motor of the attracted-iron type under simple and double excitation are also investigated. The experiments and observations described in the present paper were carried out in the Physical Laboratory of the Indian Association for the Cultivation of Science, Calcutta, where further work on the dynamics of vibration is now in progress. One very interesting case which has been worked out is that of the Combinational vibrations of a system maintained by subjecting it simultaneously to two simple harmonic forces varying its spring. 'This is experimentally realized by attaching a stretched string at its two extremities to the prongs of two tuning-forks of different periods, the directions of motion of which are parallel to the string. If $M$ and $N$ are the frequencies of the forks, it is found that the string is set into vigorous transverse oscillation if its tension is so adjusted that the natural frequency is nearly equal to $\frac{1}{2}(\mathrm{M} m \pm \mathrm{N} n)$, where $m$ and $n$ are integers. Further details of this investigation will be published in due course. 
Raman,

Fig. 1.

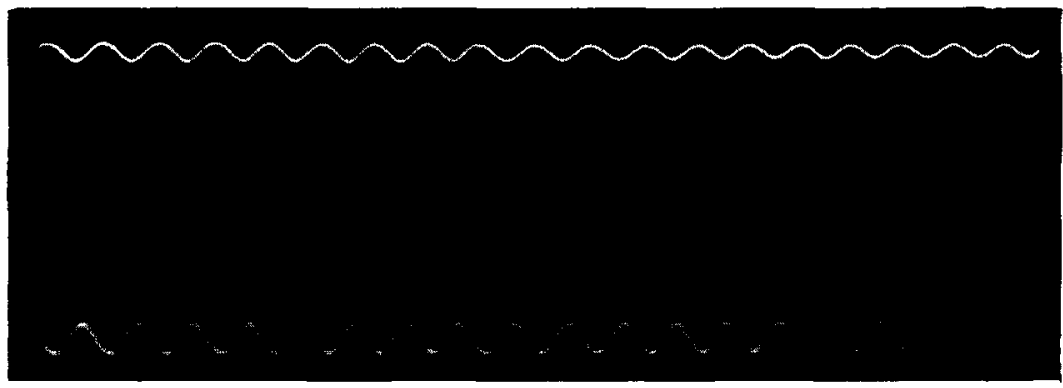

Fio. 3.

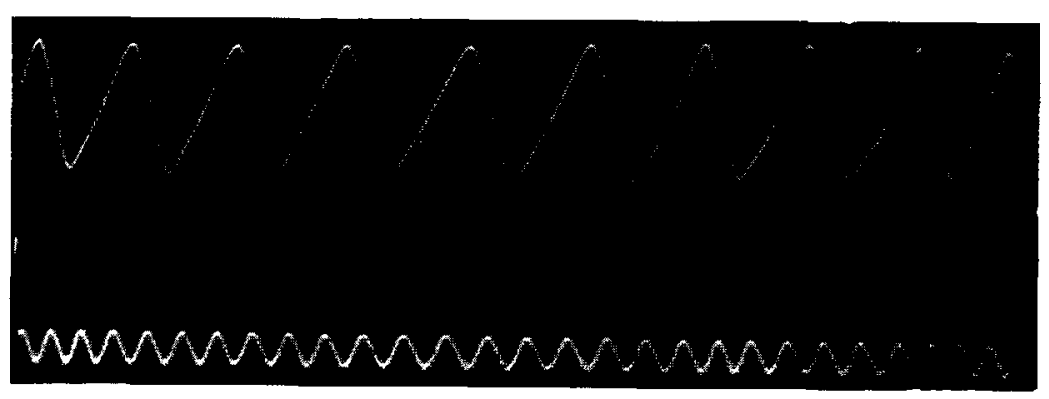

FIG. 5. 
Phil. Mag. Ser. 6, Vol. 29, Pl. II.

Fig. 2.

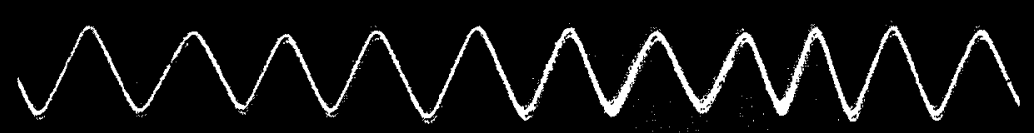

FIG. 4.

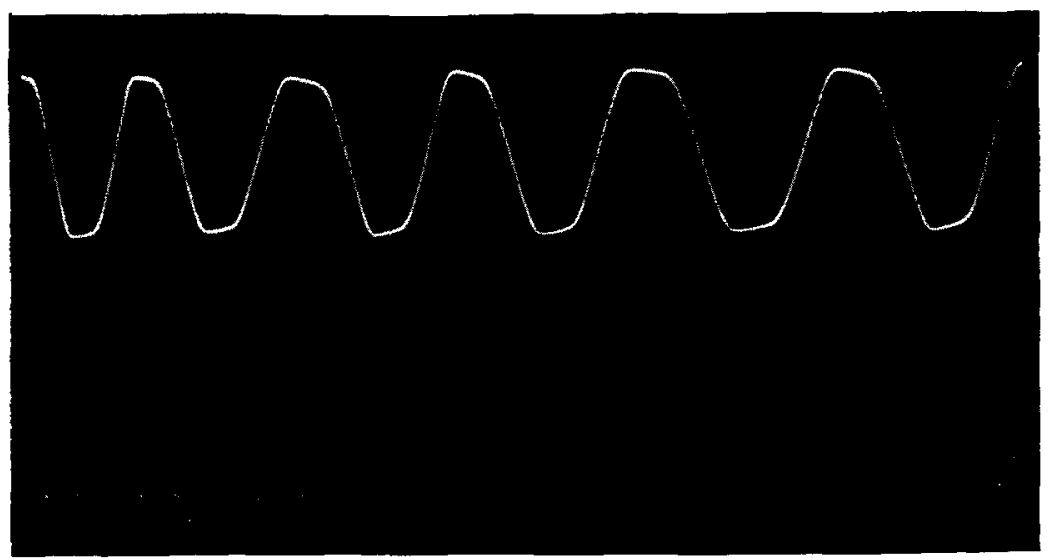

Fig. 6.

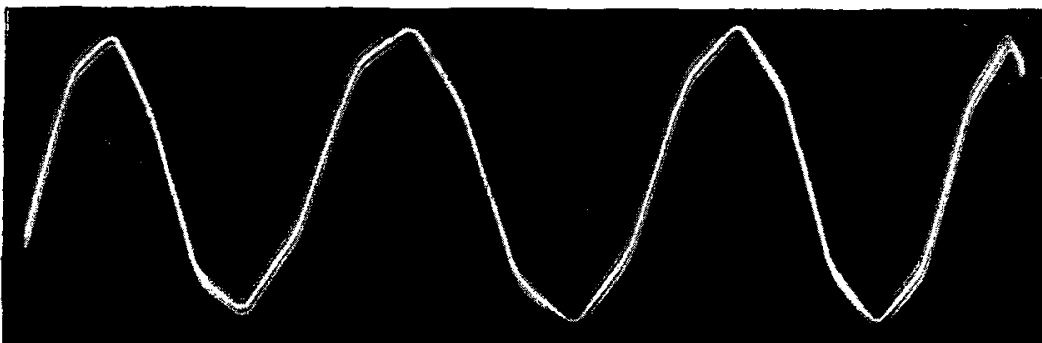

